\section{(B) OPEN ACCESS}

\title{
Alcohol and drug use during unprotected anal intercourse among gay and bisexual men in Scotland: what are the implications for HIV prevention?
}

\author{
Jessica Li, ${ }^{1}$ Lisa M McDaid ${ }^{2}$
}

\begin{abstract}
'Section of Public Health, University of Sheffield, Sheffield, UK

${ }^{2} \mathrm{MRC} / \mathrm{CSO}$ Social and Public Health Sciences Unit, University of Glasgow, Glasgow, UK
\end{abstract}

Correspondence to Dr Lisa M McDaid, MRC/CSO Social and Public Health Sciences Unit, University of Glasgow, 4 Lilybank Gardens, Glasgow, G12 8RZ, UK; lisa.mcdaid@glasgow.ac.uk.

Received 30 April 2013 Revised 4 July 2013 Accepted 4 August 2013 Published Online First 17 December 2013

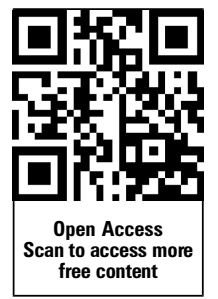

To cite: Li J, McDaid LM. Sex Transm Infect 2014;90:125-132.

\begin{abstract}
Objectives To examine alcohol and drug use during unprotected anal intercourse (UAI), and whether use is associated with HIV-related risk behaviours among gay and bisexual men in Scotland.
\end{abstract}

Methods Cross-sectional survey of 17 gay commercial venues in Glasgow and Edinburgh in May 2011

( $n=1515,65.2 \%$ response rate); 639 men reporting UAI are included.

Results $14.4 \%$ were always and $63.4 \%$ were sometimes drunk during UAI in the previous 12 months; $36.3 \%$ always/sometimes used poppers; $22.2 \%$ always/ sometimes used stimulant or other recreational/illicit drugs; and $14.1 \%$ always/sometimes used Viagra. All were significantly correlated and, in multivariate analysis, the adjusted odds of having UAI with 2+ partners in the previous 12 months were significantly higher for men who reported stimulant or recreational/illicit drug use during UAI (AOR=2.75, 95\% Cl 1.74 to 4.34) and the adjusted odds of UAI with casual partners were higher for men who reported poppers use $(A O R=1.50,95 \% \mathrm{Cl}$ 1.03 to 2.17). Men who reported always being drunk during UAI were more likely to report UAI with 2+ partners $(A O R=1.68,95 \% \mathrm{Cl} 1.01$ to 2.81$)$, casual partners (AOR=2.18,95\% Cl 1.27 to 3.73), and partners of unknown/discordant HIV status $(A O R=2.14$, $95 \% \mathrm{Cl} 1.29$ to 3.53$)$, than men who were not.

Conclusions Our study suggests alcohol and drug use may be relatively common during UAI among gay and bisexual men in Scotland. Brief alcohol or drug interventions, particularly in clinical settings, are justified, but should be properly evaluated and take into account the potential influence of broader, situational and social factors on sexual risk.

\section{INTRODUCTION}

With continuing and increasing HIV incidence among men who have sex with men (MSM) in the $\mathrm{UK},{ }^{1}$ ongoing research is required to determine which factors are associated with HIV-related sexual risk behaviours and could be amenable to intervention. The Scottish and English Sexual Health Frameworks identify MSM and those who have alcohol and drug problems as higher-risk groups for poor sexual health outcomes, ${ }^{2}{ }^{3}$ and there is growing focus on the rise in alcohol consumption and related health problems in the UK. In response to these issues, The Royal College of Physicians and British Association for Sexual Health and HIV has recommended that sexual health settings should distribute information on alcohol-related harms and could facilitate brief alcohol interventions to reduce consumption and related sexual ill health. ${ }^{4}$

Some studies have found alcohol and substance use to be significant factors in HIV acquisition among MSM, ${ }^{5}$ with reported links between alcohol and drug use and HIV-related sexual risk behaviour (such as unprotected anal intercourse (UAI) ${ }^{5}{ }^{6}$ ), HIV infection $^{78}$ and sexually transmitted infections (STI). ${ }^{9}$ Although a number of studies have focused primarily on intravenous drug use, ${ }^{10}{ }^{11}$ an emphasis on noninjecting drug use should also be considered because research suggests gay and bisexual populations are more likely to report higher use of alcohol and drugs than the general population. ${ }^{12} 13$ Despite over two decades of research, the association between substance use and HIV remains contested, and while current research is dominated by American studies, evidence within Britain is somewhat limited. ${ }^{5}{ }^{14}$ In the 2007 UK Gay Men's Sex Survey, 85\% of men reported using alcohol in the last year, $42 \%$ had used poppers, 21\% had used Ecstasy, and 21\% had used cocaine, ${ }^{15}$ and the National LGB Drug \& Alcohol Database identified alcohol, poppers and cannabis as the most commonly used drugs among MSM. ${ }^{16} \mathrm{We}$ found only one study specific to Scotland, conducted in 1998, which reported that men who used cannabis or poppers less than two hours before sex were more likely to have unprotected sex. ${ }^{17}$ Recent estimates of alcohol and drug use during sex among MSM in the UK are particularly limited, but a recent Sigma Blast report reported that one-third of participants were always, almost always, or more often than not, using poppers during sex. ${ }^{13}$ In this paper, we examine the frequency of alcohol and drug use during UAI among gay and bisexual men in Scotland, and whether alcohol and drug use during UAI is associated with particular HIV-related sexual risk behaviours.

\section{METHODS}

The 2011 MRC Gay Men's Sexual Health Survey collected anonymous, self-complete questionnaires and (Orasure) oral fluid specimens in 17 gay commercial venues (15 bars and 2 saunas) in Glasgow and Edinburgh in May 2011. This represents the majority of exclusively gay venues in each city. A form of time and location sampling was used to recruit representative samples of gay and bisexual men using the venues. Temporary fieldworkers distributed questionnaires at two different time points in the early (19.00-21.00) and late evening (21.00-23.00). No bar was visited twice in the 
same evening and at the end of the survey, each bar had been visited at both time points on each day of the week. Each sauna was visited six times over the course of the 2-week survey period; for a $2 \mathrm{~h}$ period between 17:00-19:00 on Thursdays and between 14:00-17:00 on Saturdays and Sundays. All men present or entering the venues were asked to participate; 1515 of the men approached participated in the survey $(65.2 \%$ response rate (RR)) and 1218 provided oral fluid samples (52.4\% RR). Oral fluid specimens were analysed at the West of Scotland Specialist Virology Centre (screened for anti-HIV using an enzyme immunoassay; positives rescreened, and repeat reactives confirmed using western blot). Ethical approval was granted by University of Glasgow College of Social Sciences ethics committee.

Questionnaires included demographics (age, area of residence, employment status, sexual orientation, education and frequency of gay scene use), sexual health (HIV and STI testing) and sexual behaviour (number of sexual, anal and UAI partners, partner type and HIV status) in the previous 12 months. Men were asked "Thinking about the times you had anal sex WITHOUT a condom in the last 12 months, how often were you (a) drunk on alcohol; (b) using poppers; (c) using Viagra or other erectile dysfunction drugs; (d) using stimulant drugs (eg, speed, cocaine, etc); (e) using other recreational or illicit drugs (eg, ecstasy, ketamine)", with response options of always, sometimes and never. Responses were recoded into binary drug use categories (always/ sometimes vs never) for multivariate analyses due to the small number of cases in some of the always categories.

Men who reported having no UAI in the previous 12 months $(n=810)$ and men who reported having UAI in the previous 12 months but had missing data on any of the alcohol or drug use and UAI variables $(n=66)$ were excluded from the analyses, giving a sample of 639 men. After adjusting for survey location, sexual orientation, age, survey venue, postcode, employment status, qualifications, gay scene use, HIV/STI testing, HIV treatment optimism and HIV status, when compared with excluded men in multivariate analysis, the included sample had higher odds of identifying as gay $(\mathrm{AOR}=2.44,95 \% \mathrm{CI} 1.60$ to 3.72$)$ compared with bisexual, being aged $16-25$ years $(\mathrm{AOR}=2.35,95 \%$ CI 1.63 to 3.37$)$ and $26-35$ $(\mathrm{AOR}=1.89,95 \%$ CI 1.32 to 2.70$)$ compared with $45+$ years, visiting the gay scene at least once a week $(\mathrm{AOR}=1.29,95 \% \mathrm{CI} 1.00$ to 1.67) compared with once a month or less, having an HIV or STI test in the previous 12 months (AOR $=1.34,95 \%$ CI 1.07 to 1.67), and providing an oral fluid sample and testing HIV negative $(\mathrm{AOR}=1.82,95 \%$ CI 1.38 to 2.42$)$.

$\chi^{2}$ tests were used for bivariate comparisons. Multivariate logistic regression models were used to estimate OR and 95\% CIs for HIV-related sexual risk behaviour (UAI with two or more partners, UAI with casual partners, and UAI with partners of unknown or discordant HIV status). We adjusted for factors significant at the bivariate level $(\mathrm{p}<0.05)$, (a) alcohol, (b) poppers, (c) Viagra or other erectile dysfunction drugs and (d) stimulant or recreational/illicit drugs, and any factors associated with these. Stimulant and recreational/illicit drug use categories were combined because of significant correlations found between them $\left(r_{s}(637)=0.777, p<0.001\right)$. All alcohol and drug use variables were included in the logistic regression models because of significant associations between them. Regression models were constructed using the default method (Forced Entry Method) in SPSS 15.0 for Windows.

\section{RESULTS}

\section{Sample characteristics}

The average age of participants was 31 years (range 18-68, $\mathrm{SD}=10.07)$ and the majority of men identified as gay $(94.7 \%)$;
42.6\% were surveyed in Edinburgh and 57.4\% were surveyed in Glasgow; most $(76.6 \%)$ resided in either city or its surrounding area. Degree or postgraduate education was reported by $45.6 \%$, further/vocational education by $38.6 \%$, and secondary only by $15.9 \%$. Most men were frequent visitors of the gay scene (48.5\% attended at least once a week and $24.6 \%$ attended $2-3$ times a month). The majority disagreed with, or were uncertain about, the two HIV treatment optimism statements: 'I am less worried about HIV infection now that treatments have improved'; 'I believe that new drug therapies make people with HIV less infectious' (79.3\% and $86.6 \%$, respectively). Almost two-thirds (63.1\%) reported having had either an HIV or STI test in the previous 12 months; 24 men tested HIV positive $(3.8 \%)$ and the remainder of those who provided an oral fluid sample tested HIV negative; 91 did not provide an oral fluid sample, of whom $91.2 \%$ self-reported as HIV negative. In terms of HIV-related sexual risk behaviours, $28.0 \%$ reported $2+$ UAI partners, $52.7 \%$ reported UAI with casual partners, and $55.1 \%$ reported UAI with partners of unknown/discordant status in the previous 12 months.

\section{Alcohol and drug use}

Overall, there was a high level of alcohol and drug use during UAI in the previous 12 months among this sample. Alcohol was the most frequently used drug; 497 men $(77.8 \%)$ reported ever being drunk during UAI (14.4\% reported they were always drunk, 63.4\% were sometimes drunk). Always or sometimes using poppers was reported by 232 men (36.3\%); 22 (3.4\%) always used and 210 (32.9\%) sometimes used these. Overall, 142 men (22.2\%) reported always or sometimes using stimulant, recreational or other illegal drugs. Within this category, 13 men $(2.0 \%)$ reported that they always used stimulant drugs and 98 $(15.3 \%)$ sometimes used these; 11 men $(1.7 \%)$ reported always using recreational or other illicit drugs, and 117 (18.3\%) reported sometimes using these. Viagra was the least frequently reported; only 3 men $(0.5 \%)$ reported always using it and $87(13.6 \%)$ said that they sometimes used it.

There were significant associations between the alcohol and drug use variables; $39.8 \%$ of men who used alcohol during UAI also reported using poppers compared with $23.9 \%$ of men who never used alcohol $\left(\chi^{2}=12.07, \mathrm{p}=0.001\right) ; 27.0 \%$ of men using alcohol had used stimulant or recreational/illicit drugs compared with $5.6 \%$ of men who never used it $\left(\chi^{2}=29.07, \mathrm{p}<0.001\right)$. Significant associations were also found among Viagra and other drug use. Over half of those who used Viagra reported using poppers compared with one-third of those who never used Viagra $\left(\chi^{2}=27.87, p<0.001\right)$, and half of those using Viagra also used stimulant or recreational/illicit drugs compared with one-fifth of those who never used it $\left(\chi^{2}=50.58, \mathrm{p}<0.001\right)$. Finally, those using stimulant or recreational/illicit drugs were more likely to use alcohol $\left(\chi^{2}=29.07, \mathrm{p}<0.001\right)$, poppers $\left(\chi^{2}=43.8, p<0.001\right)$, and Viagra $\left(\chi^{2}=50.58, p<0.001\right)$.

\section{Factors associated with alcohol and drug use}

Factors associated with alcohol and drug use during UAI in the previous 12 months are shown in table 1 . Younger men, those who resided in Glasgow or Edinburgh, and those who frequently visited the gay scene were more likely to report always or sometimes using alcohol. Older men and men who were HIV positive, were more likely to report always or sometimes using poppers. HIV positive men were also more likely to report always or sometimes using a stimulant or other recreational/ illicit drug during UAI. Men who were older, more optimistic about HIV treatments and HIV positive, were more likely to 
Table 1 Factors associated with alcohol and drug use during unprotected anal intercourse in the previous 12 months: $n$, row \% ( $n=639$ )

\begin{tabular}{|c|c|c|c|c|c|c|c|c|c|c|c|c|}
\hline & \multicolumn{3}{|c|}{ Drunk on alcohol } & \multicolumn{3}{|c|}{ Used poppers } & \multicolumn{3}{|c|}{$\begin{array}{l}\text { Used stimulant or recreational/ } \\
\text { illicit drugs }\end{array}$} & \multicolumn{3}{|c|}{ Used Viagra } \\
\hline & $\begin{array}{l}\text { Never, } \\
\mathrm{n}(\%)\end{array}$ & $\begin{array}{l}\text { Always/ } \\
\text { sometimes, } \\
\text { n (\%) }\end{array}$ & $p$ Value & $\begin{array}{l}\text { Never, } \\
\mathrm{n}(\%)\end{array}$ & $\begin{array}{l}\text { Always/ } \\
\text { sometimes, } \\
\text { n (\%) }\end{array}$ & $\mathrm{p}$ Value & $\begin{array}{l}\text { Never, } \\
\mathrm{n}(\%)\end{array}$ & $\begin{array}{l}\text { Always/ } \\
\text { sometimes, } \\
\text { n (\%) }\end{array}$ & $\mathrm{p}$ Value & $\begin{array}{l}\text { Never, } \\
n(\%)\end{array}$ & $\begin{array}{l}\text { Always/ } \\
\text { sometimes, } \\
\text { n (\%) }\end{array}$ & $\mathrm{p}$ Value \\
\hline Overall & $142(22.2)$ & $497(77.8)$ & & $407(63.7)$ & $232(36.3)$ & & $497(77.8)$ & $142(22.2)$ & & $549(85.9)$ & $90(14.1)$ & \\
\hline \multicolumn{13}{|l|}{ Survey location } \\
\hline Edinburgh & $66(24.3)$ & $206(75.7)$ & 0.285 & $168(61.8)$ & $104(38.2)$ & 0.383 & $216(79.4)$ & $56(20.6)$ & 0.392 & $234(86.0)$ & $38(14.0)$ & 0.943 \\
\hline Glasgow & $76(20.7)$ & $291(79.3)$ & & $239(65.1)$ & $128(34.9)$ & & $281(76.6)$ & $86(23.4)$ & & 315 (85.8) & $52(14.2)$ & \\
\hline \multicolumn{13}{|l|}{ Sexual orientation } \\
\hline Gay & $133(22.1)$ & 469 (77.9) & 0.844 & $382(63.5)$ & $220(36.5)$ & 0.621 & 472 (78.4) & 130 (21.6) & 0.284 & $520(86.4)$ & 82 (13.6) & 0.255 \\
\hline Bisexual & $8(23.5)$ & $26(76.5)$ & & 23 (67.6) & 11 (32.4) & & $24(70.6)$ & $10(29.4)$ & & $27(79.4)$ & $7(20.6)$ & \\
\hline \multicolumn{13}{|l|}{ Age (years) } \\
\hline $16-25$ & $43(18.5)$ & $189(81.5)$ & $<0.001$ & $163(70.3)$ & 69 (29.7) & 0.035 & $180(77.6)$ & $52(22.4)$ & 0.167 & $222(95.7)$ & $10(4.3)$ & $<0.001$ \\
\hline $26-35$ & $38(17.3)$ & $182(82.7)$ & & $138(62.7)$ & $82(37.3)$ & & $172(78.2)$ & $48(21.8)$ & & $196(89.1)$ & $24(10.9)$ & \\
\hline $36-45$ & $36(29.0)$ & $88(71.0)$ & & 70 (56.5) & $54(43.5)$ & & 90 (72.6) & $34(27.4)$ & & $88(71.0)$ & $36(29.0)$ & \\
\hline $45+$ & $25(40.3)$ & 37 (59.7) & & $35(56.5)$ & $27(43.5)$ & & $54(87.1)$ & $8(12.9)$ & & $42(67.7)$ & $20(32.3)$ & \\
\hline \multicolumn{13}{|l|}{ Area of residence } \\
\hline Elsewhere & $46(31.7)$ & $99(68.3)$ & 0.001 & $91(62.8)$ & $54(37.2)$ & 0.763 & $121(83.4)$ & ) $24(16.6)$ & 0.072 & $126(86.9)$ & $19(13.1)$ & 0.617 \\
\hline Glasgow/Edinburgh & $90(19.0)$ & $384(81.0)$ & & $304(64.1)$ & 170 (35.9) & & $362(76.4)$ & $112(23.6)$ & & $404(85.2)$ & 70 (14.8) & \\
\hline \multicolumn{13}{|l|}{ Employment } \\
\hline Employed & $114(22.2)$ & 399 (77.8) & 1.00 & 319 (62.2) & 194 (37.8) & 0.109 & 404 (78.8) & $109(21.2)$ & 0.232 & $437(85.2)$ & 76 (14.8) & 0.284 \\
\hline Other & $28(22.2)$ & $98(77.8)$ & & $88(69.8)$ & $38(30.2)$ & & $93(73.8)$ & $33(26.2)$ & & $112(88.9)$ & $14(11.1)$ & \\
\hline \multicolumn{13}{|l|}{ Education } \\
\hline Secondary & $20(21.1)$ & 75 (78.9) & 0.860 & $65(68.4)$ & 30 (31.6) & 0.561 & $72(75.8)$ & $23(24.2)$ & 0.837 & $81(85.3)$ & $14(14.7)$ & 0.262 \\
\hline Further/vocational & $48(20.8)$ & $183(79.2)$ & & 147 (63.6) & $84(36.4)$ & & $182(78.8)$ & $49(21.2)$ & & $206(89.2)$ & $25(10.8)$ & \\
\hline Degree/postgraduate & $62(22.7)$ & $211(77.3)$ & & $170(62.3)$ & $103(37.7)$ & & $212(77.7)$ & $61(22.3)$ & & $230(84.2)$ & $43(15.8)$ & \\
\hline \multicolumn{13}{|c|}{ Frequency of gay scene use } \\
\hline Once a month or less & $56(32.6)$ & $116(67.4)$ & $<0.001$ & $117(68.0)$ & $55(32.0)$ & 0.299 & $143(83.1)$ & $29(16.9)$ & 0.071 & $140(81.4)$ & $32(18.6)$ & 0.084 \\
\hline 2-3 times a month & $34(21.7)$ & $123(78.3)$ & & $94(59.9)$ & $63(40.1)$ & & $124(79.0)$ & $33(21.0)$ & & $141(89.8)$ & $16(10.2)$ & \\
\hline Once or more a week & $52(16.8)$ & $258(83.2)$ & & $196(63.2)$ & $114(36.8)$ & & $230(74.2)$ & $80(25.8)$ & & $268(86.5)$ & $42(13.5)$ & \\
\hline \multicolumn{13}{|c|}{ HIV treatment optimism $1^{*}$} \\
\hline Agree & $32(25.0)$ & $96(75.0)$ & 0.409 & $80(62.5)$ & $48(37.5)$ & 0.827 & $95(74.2)$ & ) $33(25.8)$ & 0.287 & $103(80.5)$ & $25(19.5)$ & 0.045 \\
\hline Uncertain/disagree & $106(21.6)$ & $385(78.4)$ & & $312(63.5)$ & $179(36.5)$ & & $386(78.6)$ & $105(21.4)$ & & $429(87.4)$ & $62(12.6)$ & \\
\hline \multicolumn{13}{|c|}{ HIV treatment optimism $2^{*}$} \\
\hline Agree & $21(25.3)$ & $62(74.7)$ & 0.479 & $53(63.9)$ & $30(36.1)$ & 0.915 & $58(69.9)$ & $25(30.1)$ & 0.066 & $65(78.3)$ & $18(21.7)$ & 0.032 \\
\hline Uncertain/disagree & $117(21.8)$ & $419(78.2)$ & & $339(63.2)$ & $197(36.8)$ & & $423(78.9)$ & $113(21.1)$ & & $467(87.1)$ & $69(12.9)$ & \\
\hline \multicolumn{13}{|c|}{ Had either an HIV or STI test in previous 12 months } \\
\hline No & $56(24.1)$ & $176(75.9)$ & 0.354 & $150(64.7)$ & $82(35.3)$ & 0.749 & $189(81.5)$ & $43(18.5)$ & 0.083 & $204(87.9)$ & $28(12.1)$ & 0.322 \\
\hline Yes & $83(21.0)$ & $313(79.0)$ & & $251(63.4)$ & $145(36.6)$ & & $299(75.5)$ & $97(24.5)$ & & $337(85.1)$ & $59(14.9)$ & \\
\hline \multicolumn{13}{|l|}{ HIV status (from saliva test) } \\
\hline HIV- & $116(22.1)$ & 408 (77.9) & 0.945 & $342(65.3)$ & $182(34.7)$ & 0.049 & $420(80.2)$ & ) $104(19.8)$ & $<0.001$ & $461(88.0)$ & $63(12.0)$ & $<0.001$ \\
\hline HIV+ & $6(25.0)$ & $18(75.0)$ & & $10(41.7)$ & $14(58.3)$ & & $11(45.8)$ & $13(54.2)$ & & $12(50.0)$ & $12(50.0)$ & \\
\hline $\begin{array}{l}\text { Did not provide oral } \\
\text { fluid specimen }\end{array}$ & $20(22.0)$ & $71(78.0)$ & & $55(60.4)$ & 36 (39.6) & & $66(72.5)$ & $25(27.5)$ & & 76 (83.5) & $15(16.5)$ & \\
\hline
\end{tabular}

have always or sometimes used Viagra. The adjusted odds of all these factors except for both HIV treatment optimism measures remained significant in multivariate analyses for all alcohol/drug use categories (data not shown).

\section{Factors associated with HIV-related sexual risk behaviour}

Tables 2-4 show factors associated with UAI with $2+$ partners, casual partners and partners of unknown/discordant status in the previous 12 months.

At the bivariate level, the likelihood of having UAI with $2+$ partners was significantly higher for men who reported always or sometimes using poppers, stimulant or other recreational/ illicit drugs, or Viagra (table 2). In multivariate analysis, the odds of reporting UAI with $2+$ partners remained significantly higher for men who reported always or sometimes using a stimulant or recreational/illicit drugs, visited the gay scene at least 2-3 times a month compared with once a month or less, and had a recent HIV or STI test. The odds were lower for men who did not provide an oral fluid specimen (table 2).

Men who reported always or sometimes using alcohol, poppers, or stimulant or recreational/illicit drugs during UAI were more likely to report UAI with casual partners than men who did not (table 3). In multivariate analysis, poppers use remained significant (table 3). The odds of UAI with casual 
Table 2 Factors associated with unprotected anal intercourse with two or more partners in the previous 12 months: $n$, row \%, unadjusted and multivariate logistic regression $(\mathrm{n}=639)$

\begin{tabular}{|c|c|c|c|c|c|c|c|}
\hline & Yes, n (\%) & OR & $95 \% \mathrm{Cl}$ & $\mathrm{p}$ Value & $\mathrm{AOR}^{*}$ & $95 \% \mathrm{Cl}$ & $\mathrm{p}$ Value \\
\hline Overall & $179(28.0)$ & & & & & & \\
\hline \multicolumn{8}{|l|}{ Survey location } \\
\hline Edinburgh & $66(24.3)$ & 1 & & & & & \\
\hline Glasgow & $113(30.8)$ & 1.39 & 0.97 to 1.98 & 0.070 & & & \\
\hline \multicolumn{8}{|l|}{ Sexual orientation } \\
\hline Gay & $164(27.2)$ & 1 & & & 1 & & \\
\hline Bisexual & $15(44.1)$ & 2.11 & 1.05 to 4.25 & 0.037 & 1.81 & 0.85 to 3.86 & 0.124 \\
\hline \multicolumn{8}{|l|}{ Aget } \\
\hline $16-25$ & $65(28.0)$ & 1 & & 0.392 & 1 & & 0.582 \\
\hline $26-35$ & $57(25.9)$ & 0.90 & 0.59 to 1.36 & & 0.96 & 0.61 to 1.52 & \\
\hline $36-45$ & $34(27.4)$ & 0.97 & 0.60 to 1.58 & & 0.90 & 0.51 to 1.59 & \\
\hline $45+$ & $23(37.1)$ & 1.52 & 0.84 to 2.73 & & 1.46 & 0.74 to 2.89 & \\
\hline \multicolumn{8}{|l|}{ Area of residence } \\
\hline Elsewhere & $32(22.1)$ & 1 & & & 1 & & \\
\hline Glasgow/Edinburgh & $143(30.2)$ & 1.53 & 0.98 to 2.37 & 0.059 & 1.18 & 0.72 to 1.91 & 0.514 \\
\hline \multicolumn{8}{|l|}{ Employment } \\
\hline Employed & $137(26.7)$ & 1 & & & & & \\
\hline Other & $42(33.3)$ & 1.37 & 0.90 to 2.09 & 0.139 & & & \\
\hline \multicolumn{8}{|l|}{ Education } \\
\hline Secondary & $25(26.3)$ & 1.06 & 0.62 to 1.80 & & & & \\
\hline Further/vocational & $73(31.6)$ & 1.37 & 0.93 to 2.02 & & & & \\
\hline Degree/postgraduate & $69(25.3)$ & 1 & & 0.441 & & & \\
\hline \multicolumn{8}{|l|}{ Frequency of gay scene use } \\
\hline Once a month or less & $29(16.9)$ & 1 & & $<0.001$ & 1 & & 0.002 \\
\hline $2-3$ times a month & $42(26.8)$ & 1.80 & 1.06 to 3.07 & & 2.00 & 1.11 to 3.61 & \\
\hline Once or more a week & $108(34.8)$ & 2.64 & 1.66 to 4.19 & & 2.60 & 1.54 to 4.39 & \\
\hline \multicolumn{8}{|l|}{ HIV treatment optimism $1 \S$} \\
\hline Agree & $44(34.4)$ & 1 & & & & & \\
\hline Uncertain/disagree & $129(26.3)$ & 0.68 & 0.45 to 1.03 & 0.070 & & & \\
\hline \multicolumn{8}{|l|}{ HIV treatment optimism $2 \S$} \\
\hline Agree & $28(33.7)$ & 1 & & & & & \\
\hline Uncertain/disagree & $145(27.1)$ & 0.73 & 0.45 to 1.19 & 0.208 & & & \\
\hline \multicolumn{8}{|c|}{ Had either an HIV or STI test in previous 12 months } \\
\hline No & $43(18.5)$ & 1 & & & 1 & & \\
\hline Yes & $130(32.8)$ & 2.15 & 1.45 to 3.18 & $<0.001$ & 1.89 & 1.24 to 2.86 & 0.003 \\
\hline \multicolumn{8}{|l|}{ HIV status (from saliva test) } \\
\hline HIV- & $150(28.6)$ & 1 & & 0.010 & 1 & & 0.003 \\
\hline $\mathrm{HIV}+$ & $12(50.0)$ & 2.49 & 1.10 to 5.67 & & 1.52 & 0.59 to 3.91 & \\
\hline Did not provide oral fluid specimen & $17(18.7)$ & 0.57 & 0.33 to 1.00 & & 0.48 & 0.26 to 0.88 & \\
\hline \multicolumn{8}{|l|}{ Drunk on alcoholø } \\
\hline Never & $35(24.6)$ & 1 & & & 1 & & \\
\hline Always/sometimes & $144(29.0)$ & 1.25 & 0.81 to 1.91 & 0.312 & 0.81 & 0.49 to 1.33 & 0.397 \\
\hline \multicolumn{8}{|l|}{ Used poppers } \\
\hline Never & $95(23.3)$ & 1 & & & 1 & & \\
\hline Always/sometimes & $84(36.2)$ & 1.86 & 1.31 to 2.65 & 0.001 & 1.44 & 0.96 to 2.14 & 0.076 \\
\hline \multicolumn{8}{|l|}{ Used stimulant or recreational/illicit drugs } \\
\hline Never & $111(22.3)$ & 1 & & & 1 & & \\
\hline Always/sometimes & $68(47.9)$ & 3.20 & 2.16 to 4.73 & $<0.001$ & 2.75 & 1.74 to 4.34 & $<0.001$ \\
\hline \multicolumn{8}{|l|}{ Used Viagra } \\
\hline Never & $138(25.1)$ & 1 & & & 1 & & \\
\hline Always/sometimes & $41(45.6)$ & 2.49 & 1.58 to 3.94 & $<0.001$ & 1.70 & 0.97 to 2.98 & 0.062 \\
\hline
\end{tabular}


Table 3 Factors associated with unprotected anal intercourse with casual partners in the previous 12 months: n, row \%, unadjusted and multivariate logistic regression $(n=639)$

\begin{tabular}{|c|c|c|c|c|c|c|c|}
\hline & Yes, n (\%) & OR & $95 \% \mathrm{Cl}$ & $\mathrm{p}$ Value & $\mathrm{AOR}^{*}$ & $95 \% \mathrm{Cl}$ & p Value \\
\hline Overall & $337(52.7)$ & & & & & & \\
\hline \multicolumn{8}{|l|}{ Survey location } \\
\hline Edinburgh & $126(46.3)$ & 1 & & & 1 & & \\
\hline Glasgow & $211(57.5)$ & 1.57 & 1.14 to 2.15 & 0.005 & 1.48 & 1.05 to 2.11 & 0.028 \\
\hline \multicolumn{8}{|l|}{ Sexual orientation } \\
\hline Gay & $311(51.7)$ & 1 & & & 1 & & \\
\hline Bisexual & $25(73.5)$ & 2.60 & 1.19 to 5.66 & 0.016 & 2.17 & 0.94 to 5.02 & 0.071 \\
\hline \multicolumn{8}{|l|}{ Age } \\
\hline 16 to 25 & $146(62.9)$ & 1 & & 0.001 & 1 & & 0.036 \\
\hline $26-35$ & $105(47.7)$ & 0.54 & 0.37 to 0.78 & & 0.62 & 0.41 to 0.94 & \\
\hline $36-45$ & $57(46.0)$ & 0.50 & 0.32 to 0.78 & & 0.58 & 0.35 to 0.96 & \\
\hline $45+$ & $28(45.2)$ & 0.49 & 0.28 to 0.86 & & 0.47 & 0.24 to 0.89 & \\
\hline \multicolumn{8}{|l|}{ Area of residencet } \\
\hline Elsewhere & $69(47.6)$ & 1 & & & 1 & & \\
\hline Glasgow/Edinburgh & $256(54.0)$ & 1.29 & 0.89 to 1.88 & 0.176 & 1.09 & 0.72 to 1.66 & 0.681 \\
\hline \multicolumn{8}{|l|}{ Employment } \\
\hline Employed & $266(51.9)$ & 1 & & & & & \\
\hline Other & $71(56.3)$ & 1.20 & 0.81 to 1.78 & 0.365 & & & \\
\hline \multicolumn{8}{|l|}{ Education } \\
\hline Secondary & $52(54.7)$ & 1.61 & 1.01 to 2.58 & & 1.42 & 0.85 to 2.36 & \\
\hline Further/vocational & $142(61.5)$ & 2.13 & 1.49 to 3.04 & & 1.90 & 1.29 to 2.81 & \\
\hline Degree/postgraduate & $117(42.9)$ & 1 & & $<0.001$ & 1 & & 0.009 \\
\hline \multicolumn{8}{|l|}{ Frequency of gay scene use } \\
\hline Once a month or less & $71(41.3)$ & 1 & & $<0.001$ & 1 & & 0.001 \\
\hline $2-3$ times a month & $74(47.1)$ & 1.27 & 0.82 to 1.96 & & 1.20 & 0.74 to 1.93 & \\
\hline Once or more a week & $192(61.9)$ & 2.32 & 1.58 to 3.39 & & 2.12 & 1.38 to 3.26 & \\
\hline \multicolumn{8}{|l|}{ HIV treatment optimism $1 \ddagger$} \\
\hline Agree & $103(44.4)$ & 1 & & & 1 & & \\
\hline Uncertain/disagree & $227(57.3)$ & 0.64 & 0.43 to 0.96 & 0.029 & 0.81 & 0.51 to 1.30 & 0.382 \\
\hline \multicolumn{8}{|l|}{ HIV treatment optimism $2 \ddagger$} \\
\hline Agree & $78(60.9)$ & 1 & & & 1 & & \\
\hline Uncertain/disagree & $246(50.1)$ & 0.61 & 0.38 to 0.99 & 0.045 & 0.52 & 0.30 to 0.92 & 0.026 \\
\hline \multicolumn{8}{|c|}{ Had either an HIV or STI test in previous 12 months } \\
\hline No & $52(62.7)$ & 1 & & & 1 & & \\
\hline Yes & $272(50.7)$ & 1.68 & 1.21 to 2.33 & 0.002 & 1.56 & 1.09 to 2.23 & 0.016 \\
\hline \multicolumn{8}{|l|}{ HIV status (from saliva test) } \\
\hline HIV- & $286(54.6)$ & 1 & & 0.044 & 1 & & 0.168 \\
\hline HIV+ & $14(58.3)$ & 1.17 & 0.51 to 2.67 & & 0.84 & 0.34 to 2.09 & \\
\hline Did not provide oral fluid specimen & $37(40.7)$ & 0.57 & 0.36 to 0.90 & & 0.62 & 0.38 to 1.02 & \\
\hline \multicolumn{8}{|l|}{ Drunk on alcohol } \\
\hline Never & $63(44.4)$ & 1 & & & 1 & & \\
\hline Always/sometimes & $274(55.1)$ & 1.54 & 1.06 to 2.24 & 0.024 & 1.19 & 0.78 to 1.83 & 0.423 \\
\hline \multicolumn{8}{|l|}{ Used poppers } \\
\hline Never & $201(49.4)$ & 1 & & & 1 & & \\
\hline Always/sometimes & $136(58.6)$ & 1.45 & 1.05 to 2.01 & 0.025 & 1.50 & 1.03 to 2.17 & 0.034 \\
\hline \multicolumn{8}{|l|}{ Used stimulant or recreational/illicit drugs } \\
\hline Never & $248(49.9)$ & 1 & & & 1 & & \\
\hline Always/sometimes & $89(62.7)$ & 1.69 & 1.15 to 2.47 & 0.007 & 1.21 & 0.77 to 1.90 & 0.421 \\
\hline \multicolumn{8}{|l|}{ Used Viagra§ } \\
\hline Never & $283(51.5)$ & 1 & & & 1 & & \\
\hline Always/sometimes & $54(60.0)$ & 1.41 & 0.90 to 2.22 & 0.138 & 1.50 & 0.86 to 2.60 & 0.150 \\
\hline
\end{tabular}


Table 4 Factors associated with unprotected anal intercourse with unknown or discordant HIV status partners in the previous 12 months: $\mathrm{n}$, row $\%$, unadjusted and multivariate logistic regression $(\mathrm{n}=639)$

\begin{tabular}{|c|c|c|c|c|c|c|c|}
\hline & Yes, n (\%) & OR & $95 \% \mathrm{Cl}$ & $\mathrm{p}$ Value & $A O R^{*}$ & $95 \% \mathrm{Cl}$ & p Value \\
\hline Overall & $352(55.1)$ & & & & & & \\
\hline \multicolumn{8}{|l|}{ Survey location } \\
\hline Edinburgh & $131(48.2)$ & 1 & & & 1 & & \\
\hline Glasgow & $221(60.2)$ & 1.63 & 1.19 to 2.24 & 0.003 & 1.67 & 1.20 to 2.33 & 0.003 \\
\hline \multicolumn{8}{|l|}{ Sexual orientation } \\
\hline Gay & $330(54.8)$ & 1 & & & & & \\
\hline Bisexual & $21(61.8)$ & 1.33 & 0.66 to 2.71 & 0.429 & & & \\
\hline \multicolumn{8}{|l|}{ Age } \\
\hline $16-25$ & $121(52.2)$ & 1 & & 0.029 & 1 & & 0.101 \\
\hline $26-35$ & $111(50.5)$ & 0.93 & 0.65 to 1.35 & & 0.89 & 0.61 to 1.32 & \\
\hline $36-45$ & 77 (62.1) & 1.50 & 0.96 to 2.35 & & 1.38 & 0.85 to 2.24 & \\
\hline $45+$ & $42(67.7)$ & 1.93 & 1.07 to 3.48 & & 1.78 & 0.94 to 3.37 & \\
\hline \multicolumn{8}{|l|}{ Area of residencet } \\
\hline Elsewhere & $81(55.9)$ & 1 & & & 1 & & \\
\hline Glasgow/Edinburgh & $260(54.9)$ & 0.96 & 0.66 to 1.40 & 0.831 & 0.93 & 0.62 to 1.38 & 0.707 \\
\hline \multicolumn{8}{|l|}{ Employment } \\
\hline Employed & $277(54.0)$ & 1 & & & & & \\
\hline Other & 75 (59.5) & 1.25 & 0.84 to 1.86 & 0.264 & & & \\
\hline \multicolumn{8}{|l|}{ Education } \\
\hline Secondary & $51(53.7)$ & 1.04 & 0.65 to 1.66 & & & & \\
\hline Further/vocational & $129(55.8)$ & 1.13 & 0.80 to 1.61 & & & & \\
\hline Degree/postgraduate & $144(52.7)$ & 1 & & 0.240 & & & \\
\hline \multicolumn{8}{|l|}{ Frequency of gay scene uset } \\
\hline Once a month or less & $88(51.2)$ & 1 & & 0.254 & 1 & & 0.151 \\
\hline 2-3 times a month & $83(52.9)$ & 1.07 & 0.69 to 1.65 & & 1.13 & 0.72 to 1.79 & \\
\hline Once or more a week & $181(58.4)$ & 1.34 & 0.92 to 1.95 & & 1.47 & 0.98 to 2.21 & \\
\hline \multicolumn{8}{|l|}{ HIV treatment optimism $1 \ddagger$} \\
\hline Agree & $72(56.3)$ & 1 & & & & & \\
\hline Uncertain/disagree & $269(54.8)$ & 0.94 & 0.64 to 1.40 & 0.767 & & & \\
\hline \multicolumn{8}{|l|}{ HIV treatment optimism $2 \ddagger$} \\
\hline Agree & 47 (56.6) & 1 & & & & & \\
\hline Uncertain/disagree & $294(54.9)$ & 0.93 & 0.58 to 1.48 & 0.762 & & & \\
\hline \multicolumn{8}{|c|}{ Had either an HIV or STI test in previous 12 months } \\
\hline No & $148(63.8)$ & 1 & & & 1 & & \\
\hline Yes & $196(49.5)$ & 0.56 & 0.40 to 0.78 & $<0.001$ & 0.52 & 0.36 to 0.73 & $<0.001$ \\
\hline \multicolumn{8}{|l|}{ HIV status (from saliva test) } \\
\hline HIV- & $285(54.4)$ & 1 & & 0.069 & & & \\
\hline HIV+ & $19(79.2)$ & 3.19 & 1.17 to 8.66 & & & & \\
\hline Did not provide oral fluid specimen & $48(52.7)$ & 0.94 & 0.60 to 1.46 & & & & \\
\hline \multicolumn{8}{|l|}{ Drunk on alcohol§ } \\
\hline Never & $75(52.8)$ & 1 & & & 1 & & \\
\hline Always/sometimes & $277(55.7)$ & 1.13 & 0.77 to 1.64 & 0.538 & 1.10 & 0.73 to 1.66 & 0.652 \\
\hline \multicolumn{8}{|l|}{ Used poppers } \\
\hline Never & $211(51.8)$ & 1 & & & 1 & & \\
\hline Always/sometimes & $141(60.8)$ & 1.44 & 1.04 to 2.00 & 0.029 & 1.27 & 0.89 to 1.82 & 0.184 \\
\hline \multicolumn{8}{|c|}{ Used stimulant or recreational/illicit drugs§ } \\
\hline Never & $264(53.1)$ & 1 & & & 1 & & \\
\hline Always/sometimes & $88(62.0)$ & 1.44 & 0.98 to 2.11 & 0.062 & 1.23 & 0.79 to 1.89 & 0.359 \\
\hline \multicolumn{8}{|l|}{ Used Viagra } \\
\hline Never & $290(52.8)$ & 1 & & & 1 & & \\
\hline Always/sometimes & $62(68.9)$ & 1.98 & 1.23 to 3.19 & 0.005 & 1.58 & 0.92 to 2.72 & 0.097 \\
\hline
\end{tabular}

*Adjusted for survey location, age, area of residence, frequency of gay scene use, HIVISTI testing, alcohol, popper, stimulant or recreational/illicit drug and Viagra use. tArea of residence and frequency of gay scene use - associated with always or sometimes using alcohol during UAI in the previous 12 months (see table 1). fHIV treatment optimism 1-'I am less worried about HIV infection now that treatments have improved', HIV treatment optimism 2-'I believe that new drug therapies make people with HIV less infectious'.

$\S$ Drunk on alcohol and used stimulant or recreational/illicit drugs-All alcohol and drug use variables were included in the logistic regression models because of significant correlations found between them. 
partners were also significantly higher among men who were surveyed in Glasgow, educated to secondary or further/vocational level compared with postgraduate, visited the gay scene at least once a week, and reported having a recent HIV or STI test. The odds were significantly lower among men aged $26+$ years and men who were uncertain or did not agree that new drug therapies make people with HIV less infectious.

The likelihood of reporting UAI with partners of unknown/ discordant HIV status was higher for men who reported always or sometimes using poppers or Viagra during UAI, but these did not remain significant in multivariate analysis (table 4). Instead, the odds were higher for men surveyed in Glasgow and lower for men who reported a recent HIV or STI test.

Given the relatively higher number of men reporting always being drunk during UAI (14.4\%) compared with always using drugs, and current concern around high alcohol consumption, a separate analysis was conducted using the always drunk category compared against sometimes or never. Men who reported always being drunk during UAI were more likely to report UAI with $2+$ partners $(\mathrm{AOR}=1.68,95 \% \mathrm{CI} 1.01$ to 2.81$)$, casual partners $(\mathrm{AOR}=2.18,95 \% \mathrm{CI} 1.27$ to 3.73$)$, or partners of unknown/discordant status $(\mathrm{AOR}=2.16,95 \% \mathrm{CI} 1.31$ to 3.56$)$ than men who sometimes or never used alcohol during UAI.

\section{DISCUSSION}

This is the first study to report on alcohol and drug use during UAI among a community-based sample of gay and bisexual men in Scotland since the late 1990s. In 1998, under half the men surveyed in Edinburgh reported alcohol use before or during sex, and less than one in 10 reported using poppers. ${ }^{17}$ In our study, more than three-quarters always or sometimes used alcohol during UAI in the previous 12 months, one-third used poppers, one-fifth used a stimulant or other recreational/illicit drugs, and one in seven used Viagra. This is comparable with general use previously reported in the 2007 UK Gay Men's Sex Survey. ${ }^{15}$ Our findings for popper use are also consistent with those from the recent Sigma Blast report in which one-third of participants reported always, almost always, or more often than not, using poppers during sex. ${ }^{13}$

However, when discussing these results there are some important limitations to our study to consider. Caution should be taken when generalising to the wider population of MSM, given this was a venue-based sample. It has been suggested that samples recruited from bars might contain higher proportions who regularly combine drug use and sex, and engage in risky sexual behaviours in general, ${ }^{18}$ so our estimates could be inflated. Furthermore, the cross-sectional nature of the data precluded any analysis of causality and our estimates could be affected by general recall or reporting bias. The anonymous nature of the survey prevents identification of men who could have completed the questionnaire more than once, but this is thought unlikely given the short timeframe of data collection and the training of fieldworkers to avoid this. General alcohol and drug use among men who did not report UAI was not collected and could not, therefore, be compared with men who reported UAI, and there were significant differences in the characteristics of the men who did and did not report UAI. Men reporting UAI, and included in our analyses, were more likely to identify as gay, were younger, more frequent scene goers, and more likely to have tested for HIV or other STIs in the previous 12 months. It is possible that there could be broader associations between substance use and sexual behaviour more generally, and this is a continuing research gap. Finally, we could not explore men's reasons for drug use. Studies have identified multiple reasons MSM decide to use alcohol or drugs including: psychological and personal (eg, child abuse, gender role confusion, homophobia, social role of alcohol and drugs), ${ }^{15}$ sexual enhancement (eg, methamphetamine and cocaine can heighten sexual desire), ${ }^{19}$ and situational factors (eg, men meeting men in clubs, bars, and other venues where drugs are available). ${ }^{19}$ Furthermore, links between alcohol and drug use and sexual risk behaviours have been widely contested because of methodological limitations which cannot control for confounding factors, such as sensation seeking ${ }^{20} 21$ or the role of social and cultural norms ${ }^{2122}$; this could not be explored in our study.

Overall, our findings on alcohol and drug use during specific sexual risk events support other recent studies, ${ }^{11} 16{ }^{17}$ and contribute to the knowledge base in this field. Polydrug use (using two or more drugs in combination) is reportedly common among MSM. ${ }^{12}{ }^{23-26}$ Here, the associations between drug use categories suggest this could be common among gay and bisexual men in Scotland reporting UAI (although we cannot confirm if drugs were actually used at the same time or at the same UAI risk event). Findings do indicate that there could be a general pattern of widespread alcohol and drug use, whether combined or not, that deserves attention.

Within our study we found that alcohol and drug use differed by age; younger men were more likely to report using alcohol during UAI, while older men were more likely to use poppers or Viagra. This is important to note because there have been fewer studies that have identified risk factors for older MSM compared with younger MSM. ${ }^{27}$ However, one US study reported older MSM were more likely to engage in higher-risk sexual activities after consuming alcohol than younger MSM. ${ }^{28} \mathrm{HIV}$ positive men were also more likely to report drug use; a finding that has similarly been reported elsewhere. ${ }^{12} 15$ This is particularly problematic because certain drugs, such as gammahydroxybutrate $(\mathrm{GHB})$ and ketamine, could potentially interfere with antiretroviral medications or adherence. ${ }^{29}$

Research on the association of alcohol and drug use to sexual risk behaviour remains somewhat mixed, and although there has been support for links between sexual risk and methamphetamine and alcohol use, particularly heavy alcohol use, findings for other drugs, such as poppers and Viagra, are more varied. ${ }^{5}$ Our results are similarly mixed. Although there was no significant association between ever using alcohol and the risk behaviours, the association we found between always using alcohol during UAI and HIV-related sexual risk behaviours supports past research on links between heavy drinking and increased sexual risk..$^{5} 2122$ While we found support for associations between stimulant, recreational or other illicit drug use and UAI with $2+$ partners, and popper use and UAI with casual partners, we did not find links between drug use and UAI with partners or unknown or discordant status or between Viagra use and any of the risk behaviours. Reasons for the differences remain unclear and of interest. Although HIV-related sexual risk behaviours were associated with one another (eg, more than two-thirds of men engaged in two or more), differential patterns of substance use may reflect differential patterns of specific risk taking within and between the sexual behaviours. This has implications for intervention development and is worthy of further qualitative investigation. Most men reporting HIV-related sexual risk behaviours also reported recent HIV/STI testing; a reassuring association we have noted previously. ${ }^{30}$ This suggests that clinical settings have access to men at high risk of reporting HIV-related behaviours, and as such, are appropriate sites for intervention, which could include a specific focus on alcohol and drug use. 
There is limited evidence of the effectiveness of interventions to reduce substance use and associated sexual risk behaviours among MSM. ${ }^{25}$ There is increasing concern about alcohol consumption and related harms to public health, and in the UK, pilot evaluations of brief alcohol interventions are underway in sexual health settings. ${ }^{3}$ However, our findings suggest the associations between alcohol and drug use and sexual risk are complex and unlikely to be amenable to straightforward intervention. Additional associations with age, gay scene use, education and location suggest the influence of broader, situational and social factors. Exploring this, and reasons for drug use (as well as polydrug use), in future research would be beneficial and enable a more in-depth understanding of the relationship between alcohol and drug use and HIV-related sexual risk behaviours; an essential step in addressing these issues in future interventions.

\section{Key messages}

- Alcohol use during UAI was common among gay and bisexual men surveyed in Scotland and substantial minorities reported use of other drugs, particularly poppers.

- Moderate correlations between the drug use categories suggest that polydrug use could be common among men in Scotland reporting UAI.

- Significant associations between alcohol and drug use during UAI and HIV-related risk behaviours were apparent, but inconsistent and mediated by other demographic and behavioural factors.

- Brief alcohol or drug interventions in clinical settings should consider the potential influence of broader, situational and social factors on sexual risk.

\section{Handling editor Jackie A Cassell}

Acknowledgements The authors thank the survey staff and fieldworkers in each city, the venue managers, their staff and all the men who agreed to participate in the survey.

Contributors $J L$ undertook analysis of data and wrote the first draft of the manuscript. LMCD designed the study and supervised and contributed to subsequent drafts. Both authors approved the final version of the manuscript.

Funding The 2011 survey was funded by the UK Medical Research Council (MRC) and Health Protection Scotland. The MRC funds JL (at the time of conducting the analysis and drafting the manuscript) and LMCD as part of the Sexual Health programme (MC_A540_5TK60) at the MRC/CSO Social and Public Health Sciences Unit, University of Glasgow.

\section{Competing interests None.}

Ethics approval University of Glasgow College of Social Sciences ethics committee.

Provenance and peer review Not commissioned; externally peer reviewed.

Data sharing statement Data sharing requests should be made directly to the study Chief Investigator, Dr Lisa McDaid.

Open Access This is an Open Access article distributed in accordance with the terms of the Creative Commons Attribution (CC BY 3.0) license, which permits others to distribute, remix, adapt and build upon this work, for commercial use, provided the original work is properly cited. See: http://creativecommons.org/ licenses/by/3.0/

\section{REFERENCES}

Phillips AN, Cambiano V, Nakagawa F, et al. Increased HIV incidence in men who have sex with men despite high levels of ART-induced viral suppression: analysis of an extensively documented epidemic. PLOS ONE 2013;8:e55312.

2 The Scottish Government. The Sexual Health and Blood Borne Virus Framework 2011-2015. Edinburgh: Scottish Government, 2011.
3 Department of Health. A Framework for Sexual Health Improvement in England. London: Department of Health, 2013.

4 Royal College of Physicians. Alcohol and sex: a cocktail for poor sexual health. Report of the Alcohol and Sexual Health Working Party. London: Royal College of Physicians, 2011.

5 Vosburgh HW, Mansergh G, Sullivan PS, et al. A review of the literature on event-level substance use and sexual risk behavior among men mho have sex with men. AIDS Behav 2012;10:1394-410.

6 Schwarcz S, Scheer S, McFarland W, et al. Prevalence of HIV infection and predictors of high-transmission sexual risk behaviors among men who have sex with men. Am J Public Health 2007;97:1067-75.

7 Buchacz K, McFarland W, Kellogg TA, et al. Amphetamine use is associated with increased HIV incidence among men who have sex with men in San Francisco. AIDS 2005; 19:1423-4.

8 Carey JW, Mejia R, Bingham T, et al. Drug use, high-risk sex behaviors, and increased risk for recent HIV infection among men who have sex with men in Chicago and Los Angeles. AIDS Behav 2009;13:1084-96.

9 Mansergh G, Purcell DW, Stall R, et al. CDC consultation on methamphetamine use and sexual risk behavior for HIV/STD infection: summary and suggestions. Public Health Rep 2006;121:127-32.

10 Degenhardt L, Mathers B, Vickerman P, et al. Prevention of HIV infection for people who inject drugs: why individual, structural, and combination approaches are needed. Lancet 2010;376:285-301.

11 Sutton AJ, House T, Hope VD, et al. Modelling HIV in the injecting drug user population and the male homosexual population in a developed country context. Epidemics 2012;4:48-56

12 Terrence Higgins Trust. MSM, drug use and sexual behaviour: Symposium report. London: CHAPS/Terrence Higgins Trust and the Centre for Research on drugs and Health Behaviour, 2010. http://www.addictioneducation.co.uk/MSM\%20Drugs\% 20and\%20Sex\%20symposium\%20report\%20FINAL.pdf (accessed 29 Apr 2013).

13 Sigma Research. The Sigma Panel Insight Blast 9: Promoting a reduction in poppers use during receptive anal intercourse. London: Sigma Research, 2012. http://www. sigmaresearch.org.uk/files/Sigma_Panel_INSIGHT_BLAST_9_Poppers.pdf (accessed 29 Apr 2013).

14 Ruf M, Lovitt C, Imrie J. Recreational drug use and sexual risk practice among men who have sex with men in the United Kingdom. Sex Transm Infect 2006;82: 95-7.

15 Keogh P, Reid D, Bourne A, et al. Wasted opportunities: Problematic alcohol and drug use among gay men and bisexual men. London: Sigma Research, 2009.

16 Buffin J, Roy A, Williams H, et al. Part of the Picture: Lesbian, gay, and bisexual people's alcohol and drug use in England (2009-2011). Manchester: The Lesbian \& Gay Foundation and the University of Central Lancashire, 2012. http://www.lgf.org. uk/documents/sep_12/FENT_1347531966_10584_POTP_Year_3_ReportFINALL. pdf (accessed 29 Apr 2013).

17 Clutterbuck DJ, Gorman D, McMillan A, et al. Substance use and unsafe sex amongst homosexual men in Edinburgh. AIDS Care 2001;13:527-35.

18 Leigh BC, Stall R. Substance use and risky sexual behavior for exposure to HIV. Issues in methodology, interpretation, and prevention. Am Psychol 1993;48:1035-45.

19 Lyons T, Chandra G, Goldstein J, et al. Breaking the bond between stimulant use and risky sex: a qualitative study. Subst Abus 2010;31:224-30.

20 Newcomb ME, Clerkin EM, Mustanski B. Sensation seeking moderates the effects of alcohol and drug use prior to sex on sexual risk in young men who have sex with men. AIDS Behav 2011;15:565-75.

21 Woolf SE, Maisto SA. Alcohol use and risk of HIV infection among men who have sex with men. AIDS Behav 2009;13:757-82.

22 Koblin BA, Husnik MJ, Colfax G, et al. Risk factors for HIV infection among men who have sex with men. AIDS 2006;20:731-39.

23 Bolding $G$, Hart $G$, Sherr $L$, et al. Use of crystal methamphetamine among gay men in London. Addiction 2006;101:1622-30.

24 Patterson T, Semple S, Zians J, et al. Methamphetamine-using HIV-positive men who have sex with men: Correlates of polydrug use. J Urban Health 2005;82:i120-6.

25 Santos GM, Das M, Colfax GN. Interventions for non-injection substance use among US men who have sex with men: what is needed. AIDS Behav 2011;15:S51-6.

26 Ostrow DG, Plankey MW, Cox C, et al. Specific sex drug combinations contribute to the majority of recent HIV seroconversions among MSM in the MACS. J Acquir Immune Defic Syndr 2009;51:349-55.

27 Heath J, Lanoye A, Maisto SA. The role of alcohol and substance use in risky sexual behavior among older men who have sex with men: a review and critique of the current literature. AIDS Behav 2012;16:578-89.

28 Mustanski B. Moderating effects of age on the alcohol and sexual risk taking association: an online daily diary study of men who have sex with men. AIDS Behav 2008;12:118-26.

29 Romanelli F, Smith KM, Pomeroy C. Use of club drugs by HIV-seropositive and HIV-seronegative gay and bisexual men. Top HIV Med 2003;11:25-32.

30 McDaid LM, Li J, Knussen C, et al. Sexually transmitted infection testing and self-reported diagnoses among a community sample of men who have sex with men in Scotland. Sex Transm Infect 2013;89:223-30. 